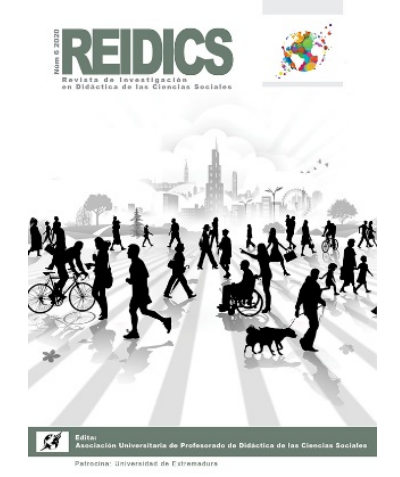

\title{
REIDICS
}

Revista de Investigación en

Didáctica de las Ciencias

Sociales
Núm. 6, 2020

Recibido 30 de junio de 2019

Aceptado 1 de octubre de 2019

E-ISSN: 2531-0968

\section{Habilidades de conciencia histórica temporal en estudiantes de formación inicial docente de Chile}

Skills of temporary historical consciousness in students of initial teacher training of Chile

Carolina Chávez Preisler

Universidad Autónoma de Barcelona

Email: carolinachavezpreisler@yahoo.es

ORCID: https://orcid.org/0000-0003-1345-5023

Joan Pagès i Blanch

Universidad Autónoma de Barcelona

Email: joan.pages@uab.cat

ORCID: https://orcid.org/0000-0001-8650-3976

DOI: https://doi.org/10.17398/2531-0968.06.24

\section{Resumen}

El presente artículo constituye una primera aproximación al análisis de resultados de una investigación doctoral en curso de la autora y que responde al objetivo de evaluar las habilidades de pensamiento histórico y su enseñanza, que poseen los estudiantes de formación inicial al comenzar la asignatura de didáctica de la historia. Para este trabajo consideramos una de las habilidades del pensamiento histórico, la conciencia histórica temporal (Santisteban, 2010; Santisteban, González y Pagès, 2010) y la analizamos en función de los siguientes aspectos: pasado, presente y futuro; articuladores temporales como cambio, continuidad, marcadores cuantitativos y cualitativos; y caracterización del tiempo histórico dependiendo si se presenta de forma lineal y/o simultánea. La metodología para abordar la investigación y dar cuenta de sus resultados es mixta con predominancia cualitativa. La muestra la constituyen 25 estudiantes pertenecientes a tres centros de educación superior en Chile. El instrumento de recogida de información es una narrativa, a través de la cual los estudiantes relataron cómo enseñarían un tema histórico. Los resultados nos permiten identificar cuáles son las fortalezas y debilidades con respecto a las habilidades de conciencia histórica temporal, caracterizar la forma como los futuros docentes piensan enseñar estas habilidades y analizar cómo se articula la historia, el tiempo y la enseñanza.

Palabras clave: pensamiento histórico; conciencia histórica temporal; temporalidad; tiempo histórico; formación inicial docente 


\begin{abstract}
This article is a first approach to the analysis of the results of an ongoing doctoral research of the author. It responds to the objective of assessing the skills of historical thinking and its teaching, which have the students of initial training at the beginning of the subject of didactics of the history. For this work we consider one of the skills of historical thinking and temporal historical awareness (Santisteban, 2010; Santisteban, González and Pagès, 2010). The following aspects were analyzed: past, present and future; temporary articulators such as change, continuity, quantitative and qualitative markers; and characterization of historical time depending on whether it is presented in a linear and / or simultaneous manner. The methodology to approach the investigation and give an account of its results is mixed with a qualitative predominance. The sample consists of 25 students belonging to three higher education centers in Chile. The instrument for collecting information is narrative, in which the students told how they would teach a historical topic. The results allow us to identify what are the strengths and weaknesses with respect to temporary historical consciousness skills, characterize the way future teachers think about teaching these skills and analyze how history, time and teaching are articulated.
\end{abstract}

Keywords: historical thinking, temporary historical consciousness, temporality, historical time, initial teacher training.

\title{
1. Introducción
}

Pagès y Santisteban (2018) reflexionan respecto a la enseñanza y al aprendizaje de la historia relacionándolos con la formación ciudadana y democrática en el contexto actual. Reconocen que la enseñanza de la historia es compleja y que sus propósitos fundamentales deberían estar orientados al desarrollo del pensamiento y la conciencia histórica; a la utilización crítica y creativa de las evidencias y/o fuentes y a la diferenciación entre argumentos racionales y sentimientos provocados por una situación vivida, identificando las falsas verdades. En este escenario se plantean la siguiente pregunta “ ¿qué formación recibe el profesorado para enseñar historia en el complejo mundo de la práctica escolar?" (Pagès y Santisteban, 2018, p.12). Esta pregunta se relaciona directamente con nuestra investigación y se complementa con otras interrogantes, por ejemplo, ¿qué habilidades de pensamiento histórico han desarrollado los y las estudiantes de formación inicial?

En Chile, el currículo de formación inicial docente está orientado a la formación disciplinar. Chávez y Pagès (2017) en un artículo donde analizan las mallas curriculares de ocho centros universitarios que imparten pedagogía en educación secundaria de historia, geografía y ciencias sociales, concluyen que la historia es la asignatura con mayor presencia en los currículos de formación inicial docente, que la didáctica tiene una distribución desigual en los centros universitarios estudiados y que, además, tiene poca presencia en la formación universitaria.

Este antecedente constituye una parte importante del problema que subyace a la actual investigación. El problema se traduce en que los centros de enseñanza superior forman a los futuros docentes priorizando las ciencias de referencia, fundamentalmente la historia y la geografía. Dando por hecho que el estudiante debe poseer habilidades de pensamiento histórico, sin generar instancias explicitas que permitan adquirirlas en caso de que no las hayan logrado 
completamente con anterioridad a la educación superior. Como plantea Domínguez (2015), esto ocurre porque se cree que se adquieren de forma natural.

La didáctica, como ciencia de referencia, está orientada a generar instancias para que los futuros docentes fortalezcan el desarrollo de sus habilidades de pensamiento histórico y luego puedan transferirlas a sus estudiantes. No obstante, en la realidad de Chile la didáctica tiene una presencia acotada en la formación inicial docente, lo que nos lleva a preguntarnos ¿están preparados los futuros docentes para desarrollar el pensamiento histórico en sus estudiantes?

Nos parece relevante dar respuesta a las preguntas planteadas mediante una investigación sistemática y rigurosa, puesto que vincular el pensamiento histórico y la formación inicial docente, es una necesidad que emerge del marco curricular vigente en Chile para la enseñanza de las ciencias sociales. En las bases curriculares se explicita que los y las estudiantes "logren desarrollar el pensamiento histórico, es decir, que comprendan que la experiencia de vivir en sociedad esta contextualizada en el tiempo, y que, en tanto seres humanos, nuestra existencia se desenvuelve en el devenir de la historia" (MINEDUC, 2015, p. 176). En consecuencia, el o la docente es el responsable de la enseñanza del pensamiento histórico y debe estar preparado para ello. Investigar las relaciones entre pensamiento histórico y formación inicial docente nos permite evaluar las habilidades de pensamiento histórico que poseen los futuros docentes, identificando aquellas que es preciso profundizar. De esta manera pretendemos contribuir a la coherencia entre la formación inicial docente y las necesidades curriculares.

Este artículo constituye una primera aproximación al análisis de resultados de una investigación doctoral en curso de la autora ${ }^{1}$ junto a su director y que responde al objetivo de evaluar las habilidades de pensamiento histórico que poseen los estudiantes de formación inicial al comenzar la asignatura de didáctica de la historia.

\section{Conciencia histórica: situarnos en el tiempo y en el espacio, siendo seres conscientes de nuestra historicidad}

La conciencia histórica convoca a diferentes disciplinas, entre ellas la historia. Carr (1978) la considera como parte esencial de la historia moderna "la historia moderna comienza cuando despiertan más y más hombres a la conciencia social y política, cuando más y más hombres toman conciencia de sus grupos respectivos como entidades históricas que tienen un pasado y un futuro" (p. 203).

Heller (1982) relaciona la conciencia histórica con las respuestas a preguntas fundamentales que se han planteado diferentes disciplinas y que orientan el desarrollo de la ciencia histórica “ ¿de dónde venimos, quiénes somos, adónde vamos? la llamaremos "conciencia histórica”, mientras que las respuestas, constituirán los estadios de la conciencia histórica”. (p.14).

Rüsen (1992) desarrolla la idea de conciencia histórica y se refiere a ella como la actividad mental del individuo mediada por la memoria y que trae al presente la experiencia del pasado, con el objetivo de comprender ese presente y que las perspectivas futuras puedan desarrollarse conforme a las experiencias pasadas invocadas en el presente. Posteriormente Rüsen (2007),

\footnotetext{
${ }^{1}$ Investigación desarrollada en el contexto de una beca doctoral en el extranjero financiada por CONICYT
} 
reforzando la dependencia y bidireccionalidad pasado/presente en la idea de conciencia histórica, plantea que la conciencia histórica representa el pasado en una interrelación explícita con el presente, guiada por conceptos de tiempo; puntualizando que la conciencia histórica esta mediada por el presente y el pasado, proyectándose al futuro.

La conciencia histórica se configura, a nuestro entender, como una cualidad del ser humano que le permite situarse en el tiempo y en el espacio y ser consciente de su historicidad. El desarrollo de la conciencia histórica requiere del aprendizaje de una serie de habilidades complejas propias del pensamiento histórico como la temporalidad, el cambio, la causalidad, la perspectiva histórica, la empatía, la utilización de fuentes y el significado histórico de los hechos o los procesos estudiados. Existen modelos que exponen, justifican y desarrollan estrategias para la enseñanza y el aprendizaje de estas habilidades ${ }^{2}$.

La interrelación entre la conciencia histórica y pensamiento histórico ha hecho que algunos autores hablen de conciencia histórica temporal. Para Santisteban (2010) la conciencia histórica temporal se configura a partir de las relaciones que se establecen entre el pasado, el presente y el futuro, permitiendo la valoración de cambios y continuidades, el progreso y la decadencia, y relacionándose con la gestión del tiempo histórico. De esta concepción de conciencia histórica temporal, hemos identificado indicadores que nos permiten analizar qué habilidades poseen los estudiantes de formación inicial del profesorado y cómo las proyectan hacia la enseñanzaaprendizaje.

\section{Temporalidad humana: lo que fuimos, lo que somos y ¿lo que seremos?}

Pasado, presente, futuro constituyen las tres categorías de la temporalidad humana (Pagès, 1988; Santisteban, 2010; Torres, 2001a). Estas categorías forman el cuerpo de la conciencia histórica temporal y son articuladas por diferentes operadores temporales como cambiocontinuidad, simultaneidad, cronología, periodización, datación, entre otros.

El pasado es la categoría temporal fundante de la ciencia histórica, pero ¿la historia es solo el pasado? La historia no es solo el pasado, es la interrelación entre las tres categorías temporales pasado, presente y futuro.

El pasado para Fontana (2013) está en las fuentes, en las evidencias, esperando que el historiador haga su trabajo y le dé forma a través de la narrativa. El "pasado explica el presente, como ocurre en toda visión de la historia, pero no lo legítima” (p.151). En palabras de De Certau (2006), la historia se hace desde el presente en retrospectiva hacia el pasado; el pasado se transforma de esta manera en el otro. El pasado como "el otro" se convierte en algo diferente que permite visualizar el cambio, significar lo "otro" y abrir paso al porvenir. En este sentido el pasado es diferente al presente, pero también el presente conserva vestigios del pasado, desarrollándose la articulación sincrónica y diacrónica entre el cambio y la continuidad.

El presente para Pagès y Santisteban (1999) "es el instante descrito por la filosofía y el acontecimiento estudiado por la historia, en realidad estos dos conceptos tienen el mismo

\footnotetext{
2 Por ejemplo, las investigaciones y artículos publicados por Santisteban, 2010; Santisteban, González y Pagès, 2010 y las investigaciones de Seixas \& Peck, 2004; Seixas, Morton, Colyer \& Fornazzary, 2013; Seixas \& Ercikan, 2015.
} 
significado, el presente es en los dos casos el punto de intersección entre el pasado y el futuro". (p. 202). Para Fontana (2013) el estudio del presente en la historia tiene un componente social enfocado a la formación ciudadana, ya que se requiere una "visión crítica del presente que explique correctamente las razones de la pobreza, el hambre y el paro, y que nos ayude a luchar contra la degradación de la naturaleza, el militarismo, la amenaza atómica, el racismo y tantos otros peligros". (p.262).

El futuro es una categoría temporal controvertida desde la historiografía, pero reconocida y valorada por su utilidad para proyectar nuestra sociedad (Braudel, 1959; Carr, 1978; Burke, 1993; Fontana, 2013). Desde la didáctica el futuro emerge como una línea de investigación y se destaca la importancia de su enseñanza, vinculándolo además con la formación ciudadana y democrática (Santisteban y Anguera, 2014).

Hemos caracterizado cada una de estas categorías temporales por separado. Sin embargo, para el desarrollo de la conciencia histórica y de la conciencia histórica temporal, es imprescindible la articulación de estas dimensiones. De Certau (2006) señala, de cara a la interrelación temporal, que una característica de la historia es "la composición de un lugar que establece en el presente la figuración ambivalente del pasado y del futuro" (p.98). Braudel (1959) hace alusión a lo que implica establecer esta relación, “(...) esclarecer de alguna manera el conocimiento del tiempo presente $y$, por tanto, necesariamente del futuro, puesto que el tiempo de hoy sólo es comprensible vinculado al tiempo del mañana. (p.130).

Refiriéndose a la enseñanza de la temporalidad en el aula. Pagès (2012b) hace referencia a que es importante que los estudiantes piensen el presente como un producto del pasado y se sientan protagonistas del futuro que pueden construir. Pagès y Santisteban (1999) señalan que:

(...) pasado, presente y futuro deben recibir atención en la enseñanza, si relegamos alguna de las tres categorías temporales, condicionamos decisivamente la reconstrucción del tiempo y limitamos el enriquecimiento de los esquemas de conocimiento al alumnado. (p. 201).

Respecto a la enseñanza del tiempo histórico y las interrelaciones entre las categorías temporales, Pagès (2012a) sostiene que enseñar el presente es más complejo que enseñar el pasado, sobre todo cuando se trata de cuestiones vivas o ideológicamente diferentes. Santisteban (2007) también señala que el pasado explica el presente, pero que en la práctica no se establecen los vínculos adecuados.

\section{Cambio y continuidad: lo que ya no es, lo que sigue siendo y lo que podría volver a ser o no ser}

El cambio tal vez sea el concepto más importante del aprendizaje de la historia, porque involucra habilidades como interpretación, explicación e intencionalidad (Pagès y Santisteban, 1999; Santisteban, 2012).

El cambio es la modificación, la transformación, la alteración o la desaparición de un statu quo. Puede ocurrir y/o repercutir en uno o más ámbitos, social, político, económico, cultural. Afectar a muchas o pocas personas, en espacios particulares o globales. Los ritmos y las duraciones del cambio son diversas. Está asociado indisolublemente a la continuidad, 
simplemente, porque lo que no cambia continua. Es un concepto múltiple, variado y complejo, constituyente fundamental de la historia y su enseñanza. Santisteban y Pagès (1999) desarrollan un modelo conceptual para comprender la complejidad del cambio. Braudel (1950) se refiere a la complejidad del cambio en relación con las estructuras:

(...) las civilizaciones son mortales en sus floreceres más exquisitos; cierto, resplandecen y después se apagan para volver a florecer bajo otras formas. Pero estas rupturas son más escasas, más espaciadas, de lo que se suele creer. Y, sobre todo, no lo destruyen todo por igual. Quiero decir que en un área determinada de civilización el contenido social puede renovarse por entero dos o tres veces sin por ello alcanzar ciertos rasgos profundos de estructura que permanecerán como poderosos distintivos de las otras civilizaciones vecinas. (p. 30).

Para Domínguez (1989). Los elementos asociados al cambio como tiempo, evolución, desarrollo, retroceso, continuidad, son esenciales para estar históricamente educados, el autor utiliza este término para referirse al pensamiento histórico.

Pagès y Santisteban (1999) y Santisteban (2007) asocian al cambio elementos como la corta o la larga duración, la coyuntura o la estructura, los ciclos y las crisis, elementos que constituyen su propuesta para el marco conceptual del cambio. Ambos autores señalan la necesidad de elaborar un modelo conceptual del cambio y del tiempo histórico para que los docentes los puedas tener en cuenta en el proceso de enseñanza y para que estos elementos se consideren en la elaboración de curriculum de historia y ciencias sociales.

La continuidad, como hemos afirmado anteriormente, está indisolublemente asociada al cambio, puesto que es lo que permanece y se mantiene, esquivando los efectos del cambio. Es complejo encontrar en la literatura que hemos revisado indicaciones que hagan alusión solo a la continuidad, puesto que siempre está asociada al cambio. Respecto al cambio y la continuidad, Domínguez (2015) propone una serie de destrezas y ámbitos a evaluar para la comprensión del tiempo, entre ellos aborda la dimensión cambio/ continuidad.

Torres (2001a) se refiere a la continuidad con el adjetivo de permanencia y en lo que él llama la arquitectura temporal, ubica al cambio y la continuidad en la categoría de operadores temporales secundarios, señalando que "la periodización colabora en la comprensión de los cambios y permanencias de los sistemas o procesos estudiados" (p.68). Explicitando que los cambios son más fáciles de identificar y de aprender que la continuidad, puesto que esta última requiere un mayor nivel de conciencia y abstracción.

\section{Articuladores temporales: datación, cronología, simultaneidad}

Mattozzi, 2000 (como se citó en Torres, 2001b) señala que en la red temporal coexisten diferentes operadores temporales: sucesión, simultaneidad, contemporaneidad, duración, ritmo, periodización. Para efectos de esta investigación y pensando en aquellos articuladores que son incorporados más frecuentemente en las narrativas que hemos analizado consideramos la datación, la cronología y la simultaneidad. Torres (2001a) establece tres categorías (primaria, secundaria y terciaria) respecto a los operadores temporales: la datación, la cronología y la simultaneidad corresponden a los articuladores temporales primarios. 
La datación constituye un operador que nos permite ubicar cronológicamente los procesos, hechos y períodos. Ayuda a desarrolla nociones de sucesión y de simultaneidad. La datación convencional incorpora años, siglos y milenios. (Pagès, 2012a).

La cronología computa los tiempos y ordena los sucesos. Está constituida fundamentalmente por la datación. Generalmente la temporalidad se asocia mayoritariamente a la cronología. Su función es enmarcar temporalmente los fenómenos históricos (Pagès, 1989). También Santisteban (2007) propone que "es necesario dar a la temporalidad la importancia que requiere y no limitarla a la cronología" (p.26).

La simultaneidad es lo que ocurre en el mismo punto de datación en espacios diferentes. Pagès y Santisteban (2010). En una propuesta de enseñanza del tiempo histórico, proponen para enseñar la simultaneidad "Buscar los elementos de simultaneidad que pudieran existir entre la historia personal y los hechos históricos a nivel local o nacional. Se puede comenzar por indagar lo que pasaba en su entorno en el año de su nacimiento". (p.303).

\section{Tiempo Histórico: la alternancia lineal y cíclica}

La historiografía tradicional basada en el paradigma positivista concibe la historia como un cumulo de acontecimiento, donde la datación y la cronología cobran gran relevancia. En contraposición a la historiografía tradicional situamos la nueva historia. Burke (1993) explica cómo el tiempo histórico es concebido desde la nueva historia. El tiempo histórico trata el análisis de las estructuras, requiere de la datación y la cronología, pero además se articula con elementos como cambio, continuidad, ritmos, estructuras, coyunturas. Braudel (1950) proporciona un ejemplo histórico basado en una crisis económica que afecto a Florencia entre 1580 y 1585 . En su relato histórico incluye todos los elementos anteriores, y pone en evidencia la complejidad que implica el tiempo histórico. Pagès (1989), precisa que la cronología es esencial para la comprensión del tiempo histórico, pero pensar únicamente que la cronología es el tiempo histórico, es errado.

El tiempo histórico en la enseñanza de la historia debe transitar entre el tiempo rotativo (cíclico) y lineal, incorporando la complejidad que nos propone Braudel (1950) con el ejemplo de Florencia. Considerando que la dialéctica pasado, presente, futuro es el sentido de la temporalidad (Pagès, 1989). Vinculando elementos de linealidad temporal en concepciones cíclicas, y viceversa (Torres, 2001b).

\section{Metodología}

La metodología de la investigación es mixta con peso cualitativo (Hernández, Fernández y Baptista, 2003). Se define en estos términos porque el paradigma en el que se ampara es el humanista interpretativo, el instrumento de recogida de información es una narrativa y los resultados se exponen de forma cualitativa y cuantitativa.

La muestra está constituida por 25 estudiantes de formación inicial que cursan al 2018 entre $5^{\circ}$ y $7^{\circ}$ semestre, cuya edad promedio es de 22 años. Un 64\% (16) son mujeres y un 36\% (9) hombres. Pertenecientes a tres universidades chilenas, una de ellas ubicada en la zona central (8 
estudiantes) y dos en la zona sur (8 y 9 estudiantes). El nombre de los estudiantes ha sido codificado con una letra y un número, ejemplo A1.

El instrumento de recogida de información fue una narrativa a través de la cual los y las futuros (a) docentes relataron cómo harían una clase de historia de síntesis, cuyo contenido decidió libremente cada estudiante. La especificación de que sea una clase de síntesis, se fundamenta en la posibilidad de incorporar todas las habilidades de pensamiento histórico en el relato. La libre elección del tema y la alternativa de que, además, el estudiante pueda consultar internet si lo estima conveniente, es relevante porque no nos interesa identificar si el estudiante sabe o no contenidos factuales, sino cuáles son sus habilidades de pensamiento histórico.

El análisis de la información se llevó a cabo a partir de la confección de categorías que emergen de los modelos de pensamiento histórico de Seixas \& Peck (2004) y Santisteban, González y Pagés (2010) y en particular de la concepción de conciencia histórica temporal de Santisteban (2010). Además del análisis categorial llevamos a cabo un análisis de contenido, que no permitió identificar la presencia o ausencia de cada indicador. La presencia de cada indicador fue valorada con un número 1 y la ausencia con un 0 , esto nos ha permitido reducir la información cualitativa y presentarla a través de porcentajes (Strauss y Corbin, 2002).

\section{Resultados y discusión}

El 100\% de los estudiantes considera el pasado en su narrativa histórica. De ellos un 96\% considera el pasado como el tiempo predominante, aunque hacen referencias al presente mencionando alguna situación o estableciendo relaciones pasado/presente o presente/pasado en diferentes niveles que hemos establecido como básico, intermedio y avanzado. Respecto al presente, un $56 \%$ del $100 \%$ de la muestra incorpora en su relato esta dimensión temporal, considerando diferentes niveles de profundidad.

Un 16\% (4) de los estudiantes establecen su relato preferentemente desde el pasado y consideran el presente en un nivel básico, por ejemplo, A3 diseña una clase cuyo tema corresponde a la República Liberal en Chile (1861-1891) e incorpora el presente como se expone a continuación:

Se irán presentando las características generales de cada presidente (desde José Joaquín Pérez hasta José Manuel Balmaceda) y las obras más características de la época y las que trascenderían en el tiempo. (A3)

Mediante la frase "trascenderían a su tiempo", A3 incorpora el presente en un nivel básico sin explicitar hechos, procesos o instituciones del presente que se relacionen con esta época histórica.

Un 40\% (10) de los estudiantes incorporan el presente en un nivel intermedio, de ellos, 9 estudiantes basan el relato predominantemente en el pasado y solo 1 en el presente. Las diferencias fundamentales entre la categoría inicial y la intermedia están determinadas por la explicitación de hechos, procesos o instituciones del presente que se vinculan con el pasado, el establecimiento de relaciones presente/pasado o pasado/presente y la consideración de aspectos de continuidad, causas y/o consecuencia (actual). A12 diseña una clase de la Guerra Fría e incorpora el presente en un nivel intermedio: 
El objetivo de la clase es que los estudiantes comprendan lo que significó la Guerra Fría en su tiempo para las personas que lo vivieron y cómo repercutió esta guerra en nuestro mundo actual (...) recalcar que la Guerra Fría explica muchos procesos que vivimos hoy en día, como la guerra en Palestina, la situación económica y política de Cuba. Así como también explica sucesos políticos vividos recientemente como lo fueron las dictaduras de América Latina (...) cómo acabó la guerra fría, con la caída de la Unión Soviética y al mismo tiempo del bloque comunista, pero siempre considerando que es un proceso que todavía tiene repercusiones hasta el día de hoy y sigue latente en nuestras vidas (...) Los estudiantes tendrán que explicar en una plana en que hecho, conflicto, situación o proceso que se vive hoy en día se puede ver reflejada las consecuencias directas o indirectas de la Guerra Fría. (A12)

Tabla 1

Niveles de presente

\begin{tabular}{l|l} 
Nivel Básico & Incorpora la dimensión temporal del presente a través de algún concepto o idea que
\end{tabular} no es principal ni predominante en el relato. Es decir, hace una mención fugaz al presente, desarrollando el relato histórico preferentemente desde el pasado. No hay referencias a la continuidad ni a la causalidad.

\begin{tabular}{|c|c|}
\hline $\begin{array}{c}\text { Nivel } \\
\text { Intermedio }\end{array}$ & $\begin{array}{l}\text { Incorpora la dimensión temporal del presente a través de preguntas o temas de } \\
\text { análisis, estableciendo relaciones pasado/presente y/o considerando explícitamente } \\
\text { aspectos de continuidad, causa y/o consecuencia (actuales). No obstante, el tiempo } \\
\text { en el que se presenta el relato sigue siendo predominantemente el pasado. }\end{array}$ \\
\hline $\begin{array}{c}\text { Nivel } \\
\text { Avanzado }\end{array}$ & $\begin{array}{l}\text { Articula el relato a partir del presente, es decir, considera un problema o situación } \\
\text { actual, lo vincula con el pasado, lo proyecta al futuro, exponiendo situaciones } \\
\text { concretas del contenido histórico. Se establecen constantemente relaciones entre las } \\
\text { tres dimensiones temporales pasado, presente y futuro, considerando explícitamente } \\
\text { aspectos de la continuidad y de la causalidad. No existe una predominancia del pasado } \\
\text { en el relato, sino una alternancia y equilibrio entre las dimensiones temporales. } \\
\text { (Pagès, 1988; } 1989 ; 2012 \mathrm{a} \text {; } 2012 \mathrm{~b} \text {; Pagès y Santisteban 1999, 2010; Santisteban } \\
2007,2010 \text {; Santisteban, González y Pagès, 2010; Domínguez, 2015; Torres } 2001 \text { y y } \\
2001 \text { b) }\end{array}$ \\
\hline
\end{tabular}

Fuente: Elaboración propia

No existen estudiantes que hayan incorporado el presente en la dimensión avanzada, fundamentalmente porque no existe una problematización del presente que le permita al estudiante enseñar el presente a través del pasado, y en sus relatos no hay equilibrio entre las dimensiones temporales. Por ejemplo, en el caso que mencionamos anteriormente de A12, para estar en una categoría de avanzado podría haber comenzado con alguna noticia o problematización actual respecto a Palestina. Desde esta situación, podría haber remontado al pasado trabajando la Guerra Fría. Posteriormente haber vuelto al presente explicitando las relaciones con el pasado y proyectando cómo esto influye en el futuro internacional, dando cuenta de aspectos de cambio, continuidad, causa y consecuencia, realizando un planteamiento retrospectivo del presente con el pasado. Simián de Molinas (1970), expone cómo trabajar el método retrospectivo en la enseñanza de la historia, situando la enseñanza de la historia desde el presente hacia el pasado.

Solo dos estudiantes, equivalente al $8 \%$ del 100\% la muestra, consideran el futuro en su relato. A16 narra la Dictadura Militar en Chile e incluye el futuro en el nivel intermedio:

Entre las reformas que se pretende ver, está el paso hacía una economía neoliberal, los pilares de apertura económica en negociación directa con países tales como EEUU e Inglaterra, como proyecto económico de los Chicago Boys, y la reforma laboral realizada por José Piñera, para que comprendan como futuros trabajadores asalariados, las condiciones laborales en las que estarán inmersos, sobre todo por ser 
futuros trabajadores, y el desarme de la organización sindical y política de los trabajadores. (A16).

Tabla 2

Niveles de Futuro

Nivel Básico $\mid$ Incorpora la dimensión temporal del futuro a través de algún concepto o idea que no es principal, ni predominante en el relato que establece, es decir, hace una mención fugaz al futuro, desarrollando el relato histórico preferentemente desde el pasado. El pasado es la dimensión temporal predominante.

\begin{tabular}{l|l}
\hline Nivel & $\begin{array}{l}\text { Incorpora la dimensión temporal del futuro a través de preguntas o temas de análisis, } \\
\text { estableciendo relaciones pasado/presente/futuro. No obstante, el tiempo en el que se } \\
\text { presenta el relato sigue siendo predominantemente pasado. Relaciona el futuro con } \\
\text { elementos de cambio, continuidad o consecuencia. }\end{array}$ \\
\hline Nivel & $\begin{array}{l}\text { No existe una predominancia del pasado en el relato, sino una alternancia y equilibrio } \\
\text { entre las dimensiones temporales pasado, presente, futuro. El presente actúa como } \\
\text { articulador, es decir, considera un problema o situación actual, lo vincula con el pasado, } \\
\text { lo proyecta al futuro, exponiendo situaciones concretas del contenido histórico. } \\
\text { Constantemente expone relaciones entre las tres dimensiones temporales pasado, } \\
\text { presente y futuro, considerando explícitamente la vinculación con elementos de la } \\
\text { formación para la ciudadanía. Relaciona el futuro con elementos de cambio, } \\
\text { continuidad o consecuencia. (Pagès, 1988; 1989; 2012a; 2012b; Pagès y Santisteban } \\
1999,2010 ; \text { Santisteban 2007, 2010; Santisteban, González y Pagès, 2010; Santisteban } \\
\text { y Angueras, 2014; Domínguez, 2015; Torres 2001a y 2001b) }\end{array}$
\end{tabular}

Fuente: Elaboración propia

Solo 14 estudiantes (56\%) de la muestra incorporan en el relato dos dimensiones temporales pasado y presente, pero no todos establecen relaciones entre las dimensiones temporales. Solo 10 de ellos (40\%), incorporan esta habilidad considerando dos dimensiones, el pasado y el presente. En cambio, no explicitan la relación con el futuro.

Tabla 3

Niveles de relación temporal

\begin{tabular}{|c|c|}
\hline Nivel Básico & $\begin{array}{l}\text { Relaciona el pasado y el presente o el presente y el pasado, a partir de una idea general. } \\
\text { La relación se desarrolla solo una o dos veces en el relato }\end{array}$ \\
\hline $\begin{array}{l}\text { Nivel } \\
\text { Intermedio }\end{array}$ & $\begin{array}{l}\text { Relaciona el pasado y el presente o el presente y el pasado, a través de un tema } \\
\text { declarado, actual y contingente. La relación se desarrolla solo una o dos veces, } \\
\text { articulando con uno o más aspectos de causa, consecuencia, cambio, continuidad. }\end{array}$ \\
\hline $\begin{array}{l}\text { Nivel } \\
\text { Avanzado }\end{array}$ & $\begin{array}{l}\text { Relaciona el pasado/presente, el presente/pasado, el presente/futuro y/o el } \\
\text { pasado/futuro, a través de un tema actual. La relación se genera en más de dos } \\
\text { oportunidades en el relato, es decir, pasado, presente, futuro articulándose } \\
\text { constantemente, a través de las causas o las consecuencias, o del cambio y la } \\
\text { continuidad. (Pagès, 1988; 1989; 2012a; 2012b; Pagès y Santisteban 1999, 2010; } \\
\text { Santisteban 2007, 2010; Santisteban, González y Pagès, 2010; Domínguez, 2015; Torres } \\
\text { 2001a y 2001b) }\end{array}$ \\
\hline
\end{tabular}

Fuente: Elaboración propia

Las relaciones temporales presente/pasado se exponen en uno (4\%) de los 10 relatos. En él se parte del relato de un fenómeno contingente desde el presente y se recurre al pasado para explicarlo. Los 9 (36\%) relatos restantes establecen la relación pasado/presente, parten de un acontecimiento histórico del pasado y recurren al presente para explicar sus causas y consecuencias, el cambio o la continuidad. 
El nivel básico de relaciones temporales pasado/presente es expuesto por un estudiante (4\%), A9, en el tema de la Guerra fría en Chile y el mundo, haciendo referencia al presente y relacionándolo con el pasado en un nivel inicial. En el cierre de la clase solicita que los alumnos, que:

(...) reflexionen, respecto a lo que sucede actualmente con los países involucrados en este conflicto según los medios de comunicación y que entienden ellos. (A9).

El nivel intermedio de relaciones temporales pasado/presente es expuesto por 9 estudiantes (36\%). A22 narra el proceso de Dictadura Militar en Chile:

(...) entregaría una guía con algunos extractos de diarios de la época (...) La actividad tiene como propósito (...) analizar profundamente lo expuesto e ir generando (...) opiniones en torno a lo planteado, para que logren comprender como se fueron dando los diversos sucesos acaecidos durante la dictadura, y cómo en ese período la vida en su totalidad de la población cambio, pues no solamente se dieron violaciones de los DDHH, o personas desaparecidas, sino que todo aquello vino acompañado de la fundación de un nuevo modelo de desarrollo económico, que generó consecuencias las cuales se plasman hasta la actualidad (...) . Cómo todo aquello repercutió en lo que vendría a ser la transición de la democracia y los diversos problemas y conflictos que afectan actualmente el país (A22)

La incorporación del cambio y la continuidad se presenta de forma muy diversa, como exponemos en la Figura 1.

\section{CAMBIO - CONTINUIDAD}

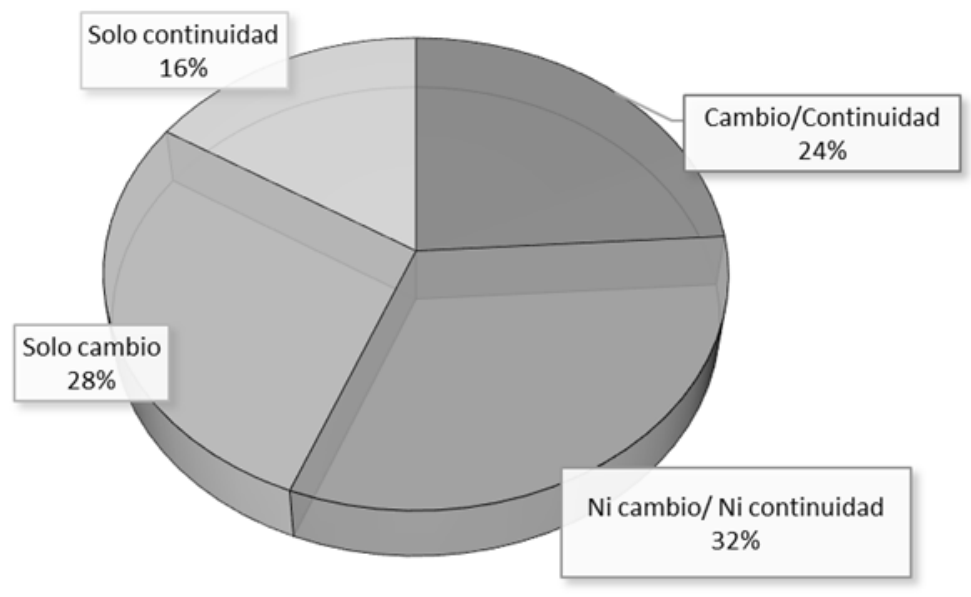

Figura 1. Incorporación del cambio y la continuidad

Hemos identificado tres categorías respecto a la incorporación del cambio y/o la continuidad en las narrativas históricas: quienes incorporan el cambio y la continuidad, 6 estudiantes (24\%); quienes solo incorporan el cambio 7 estudiantes (28\%) y quienes solo incorporan la continuidad, 4 estudiantes (16\%). Cada una de estas categorías se expresa en diferentes niveles. 
Tabla 4

Niveles de continuidad

\begin{tabular}{l|l} 
Nivel Básico & $\begin{array}{l}\text { Identifica implícitamente algunos aspectos de continuidad que corresponden al } \\
\text { período, estableciendo elementos de continuidad de corto plazo (entre dos períodos } \\
\text { consecutivos) y a largo plazo (entre períodos dispares). }\end{array}$ \\
\hline $\begin{array}{l}\text { Nivel } \\
\text { Intermedio }\end{array}$ & $\begin{array}{l}\text { Identifica explícitamente algunos aspectos de continuidad que corresponden al período, } \\
\text { estableciendo elementos de continuidad de corto plazo (entre dos períodos } \\
\text { consecutivos) y a largo plazo (entre períodos dispares). }\end{array}$ \\
\hline Nivel & $\begin{array}{l}\text { Identifica y explica explícitamente todos los elementos de continuidad que } \\
\text { corresponden al período, distinguiendo entre aquellos elementos de la continuidad que } \\
\text { se desarrollan a corto plazo (entre dos períodos consecutivos) y a largo plazo (entre } \\
\text { períodos dispares). (Pagès, 1988; 1989; 2012a; 2012b; Pagès y Santisteban 1999, } \\
\text { 2010; Santisteban 2007, 2010; Santisteban, González y Pagès, 2010; Domínguez, 2015; } \\
\text { Torres 2001a y 2001b) }\end{array}$
\end{tabular}

Fuente: Elaboración propia

Los estudiantes que incorporan solo la continuidad lo hacen, en un 100\%, en nivel básico. Incluyen la continuidad a través de generalizaciones como A1 o estableciendo relaciones presente pasado, como A25:

(...) gobiernos radicales... denotando su preocupación (los tres) por la conformación del Estado de bienestar y la modernización de este (A1)

Golpe militar, violación a los derechos humanos, plebiscito, constitución de 1980 y vuelta a la democracia. Dentro de este trabajo en grupo deben explicar en qué consiste cada temática y cómo esto ha afectado de buena o mala forma al Chile actual (A25).

Es más frecuente que los estudiantes incorporen solo el cambio; un $28 \%$ de los futuros docentes lo hace en diferentes niveles.

Tabla 5

Niveles de Cambio

\begin{tabular}{|c|c|}
\hline Nivel Básico & ifica implícitamente algunos aspectos de cambio, sin describir ni explicar. \\
\hline $\begin{array}{l}\text { Nivel } \\
\text { Intermedio }\end{array}$ & Identifica explícitamente algunos aspectos de cambio, los describe y/o explica. \\
\hline $\begin{array}{l}\text { Nivel } \\
\text { Avanzado }\end{array}$ & $\begin{array}{l}\text { Identifica y explica explícitamente la mayoría de los elementos de cambio que } \\
\text { corresponden al proceso o período, enmarcándolo cuantitativa y cualitativamente, lo } \\
\text { que le permite establecer relaciones de cambio/ continuidad con otros períodos o } \\
\text { procesos consecutivos y no consecutivos al período o proceso que relata, } \\
\text { considerando al menos dos de las siguientes habilidades: } \\
\text { - Da cuenta de la intensidad del cambio (brusco, gradual o lento). } \\
\text { - Relaciona el cambio con la temporalidad pasado, presente y futuro. } \\
\text { - Relaciona el cambio con elementos de causalidad y consecuencia. } \\
\text { - Evalúa el cambio (progreso o retroceso). } \\
\text { (Pagès, 1988; 1989; 2012a; 2012b; Pagès y Santisteban 1999, 2010; Santisteban } \\
\text { 2007, 2010; Santisteban, González y Pagès, 2010; Domínguez, 2015; Torres 2001a y } \\
\text { 2001b) }\end{array}$ \\
\hline
\end{tabular}

Fuente: Elaboración propia

Un 28\% (7) de los estudiantes incluyen el cambio. Cuatro estudiantes (16\%) consideran el cambio en un nivel básico y tres (12\%) en un nivel intermedio. Los estudiantes que lo hacen en un nivel básico no explicitan el concepto de cambio en su relato, pero es posible identificar elementos históricos asociados al cambio. Por ejemplo, A4 hace referencia al proceso de ocupación de la Araucanía y señala como característica del período: "el progresivo avance 
mediante la fundación de fuertes y ciudades" (A4). La fundación de fuertes y ciudades en territorio indígena constituye un cambio gradual, de tipo político, económico y ambiental. A4 solo lo relata como una característica del período, sin dar cuenta de que es un elemento de cambio, ni lo vincula con las causas del actual conflicto mapuche, ni con las consecuencias del período de ocupación.

Tabla 6

Niveles de Cambio y Continuidad

\begin{tabular}{|c|c|c|}
\hline & Cambio & Continuidad \\
\hline Nivel Básico & $\begin{array}{l}\text { Identifica implícitamente algunos } \\
\text { aspectos de cambio que corresponden } \\
\text { al período. }\end{array}$ & $\begin{array}{l}\text { Identifica implícitamente algunos aspectos } \\
\text { de continuidad que corresponden al período, } \\
\text { estableciendo elementos de continuidad de } \\
\text { corto plazo (entre dos períodos } \\
\text { consecutivos) y a largo plazo (entre períodos } \\
\text { alejados). }\end{array}$ \\
\hline \multirow[t]{2}{*}{$\begin{array}{l}\text { Nivel } \\
\text { Intermedio }\end{array}$} & $\begin{array}{l}\text { Identifica explícitamente algunos } \\
\text { aspectos de cambio que corresponden } \\
\text { al período que relata. }\end{array}$ & $\begin{array}{l}\text { Identifica explícitamente algunos aspectos de } \\
\text { continuidad que corresponden al período, } \\
\text { estableciendo elementos de continuidad de } \\
\text { corto plazo (entre dos períodos } \\
\text { consecutivos) y a largo plazo (entre períodos } \\
\text { alejados). }\end{array}$ \\
\hline & \multicolumn{2}{|c|}{$\begin{array}{l}\text { Cuando incorporan el cambio y la continuidad en el nivel intermedio, dan cuenta de la } \\
\text { relación entre ambos. }\end{array}$} \\
\hline \multirow[t]{2}{*}{$\begin{array}{l}\text { Nivel } \\
\text { Avanzado }\end{array}$} & $\begin{array}{l}\text { Identifica y explica explícitamente la } \\
\text { mayoría de los elementos de cambio } \\
\text { que corresponden al período, } \\
\text { enmarcándolo cuantitativa y } \\
\text { cualitativamente. Establece relaciones } \\
\text { de cambio/ continuidad con otros } \\
\text { períodos consecutivos y no } \\
\text { consecutivos al período que relata, } \\
\text { considerando al menos dos de las } \\
\text { siguientes habilidades: } \\
\text { - Da cuenta de la intensidad del cambio } \\
\text { (brusco, gradual o lento). } \\
\text { - Relaciona el cambio con la } \\
\text { temporalidad pasado, presente y } \\
\text { futuro. } \\
\text { - Relaciona el cambiocon elementos de } \\
\text { causalidad y consecuencia. } \\
\text { - Evalúa el cambio (progreso o } \\
\text { retroceso). }\end{array}$ & $\begin{array}{l}\text { Identifica y explica explícitamente todos los } \\
\text { elementos de continuidad que corresponden } \\
\text { al período, distinguiendo entre aquellos } \\
\text { elementos de la continuidad que se } \\
\text { desarrollan a corto plazo (entre dos períodos } \\
\text { consecutivos) y a largo plazo (entre } \\
\text { períodos). }\end{array}$ \\
\hline & \multicolumn{2}{|c|}{$\begin{array}{l}\text { Cuando incorporan el cambio y la continuidad en el nivel intermedio y/o avanzado dan } \\
\text { cuenta de la relación entre ambos. (Pagès, 1988; 1989; 2012a; 2012b; Pagès y } \\
\text { Santisteban 1999, 2010; Santisteban 2007,2010; Santisteban, González y Pagès, 2010; } \\
\text { Domínguez, 2015; Torres 2001a y 2001b) }\end{array}$} \\
\hline
\end{tabular}

Fuente: Elaboración propia

Aquellos estudiantes que incorporan el cambio en un nivel intermedio, explicitan el concepto dando cuenta de la habilidad que están desarrollado. Por ejemplo, A3 haciendo referencia al período de República Liberal en Chile (1861-1891):

“República Liberal en Chile en los años 60’ de la época y de modo sintético se presentarán los grandes cambios que se vieron en la época” (A3) 
Un $24 \%$ de los estudiantes incorporan el cambio y la continuidad. Para dar cuenta de los resultados hemos establecido las mismas categorías de análisis presentadas previamente, agregando al nivel intermedio y avanzado la habilidad de establecer relaciones entre el cambio y la continuidad.

Los estudiantes incorporan de manera desigual en sus narrativas el cambio y la continuidad y explicitan y detallan con mayor profundidad el cambio.

Tabla 7

Niveles incorporación del cambio y la continuidad

\begin{tabular}{c|c|c} 
& Cambio & Continuidad \\
\hline A12 & Básico & Básico \\
\hline A13 & Intermedio & Básico \\
\hline A16 & Intermedio & Básico \\
\hline A21 & Intermedio & Básico \\
\hline A22 & Intermedio & Intermedio \\
\hline A24 & Intermedio & Intermedio
\end{tabular}

Fuente: Elaboración propia

A21 explica el fin de los gobiernos oligárquicos y el comienzo de los gobiernos democráticos en Chile, e incorpora el cambio en nivel intermedio:

La explicación del concepto de mediana duración, democratización del bienestar, explicando que es un proceso que se abre en 1925 y se cierra en 1973. Explicar que es un proceso de contención de las clases dominantes a las demandas sociales, si bien es cierto garantiza una ampliación de beneficios, los comportamientos de explotación siguen, pero permiten que la disputa sea legal. También se explicará el nuevo rol del Estado y se contestará con la lógica anterior, mediante la realización de cuadros comparativos hechos colectivamente en el pizarrón (A21).

El estudiante A21 expone la extensión del cambio y además los nombra: demandas sociales, ampliación de beneficios y nuevo rol del Estado, trabajando esto último a través de un cuadro comparativo con el período anterior.

Pero A21 incluye la continuidad en un nivel básico, haciendo referencia a ella de manera implícita y a través de la relación pasado/presente, expuesta en su discurso con la frase "los comportamientos de explotación siguen":

(...) democratización del bienestar, explicando que es un proceso que se abre en 1925 y se cierra en 1973. Es un proceso de contención de las clases dominantes a las demandas sociales, si bien es cierto garantiza una ampliación de beneficios, los comportamientos de explotación siguen. (A21)

Los relatos en un 92\% (23) incorporan linealmente el tiempo histórico, relatando en una escala temporal progresiva lo que ocurre. El $4 \%$ (1) comete errores temporales de contenido por lo que no es posible integrarlo a una categoría y el 4\% (1) enmarca su narración en un tiempo estático. El resto de estudiantes, 92\% (23) desarrollan un relato lineal en el que es posible identificar las categorías expuestas en Tabla 8. 
Tabla 8

Categorías de linealidad de tiempo histórico

Linealidad

Presidencialista

Contextualizan cualitativamente el período explicitando el

$12 \%$ nombre de este, utilizan referencias como siglos, décadas o años. Posterior a esta contextualización se aborda el período desde los presidentes y sus obras.

Lineal Generalizado $\quad$ Relato generalizado, utilizando marcadores temporales cuantitativos y cualitativos insuficientes. No es un relato atemporal porque incluye marcadores cualitativos 0 cuantitativos que permite situarse y dar cuenta de la progresión temporal, pero estos son insuficientes.

Lineal
Causa/Consecuencia

Relato que sigue una linealidad del tiempo histórico, pero que se centra en explicar las cusas y las consecuencias de un proceso, utilizando marcadores temporales cualitativos $\mathrm{y}$ cuantitativos.

Lineal de Períodos $\quad$ Relato descriptivo de períodos que se van sucediendo progresivamente en un marco temporal definido. Algunos incorporan línea de tiempo.

Lineal de procesos y Relato donde se identifican cualitativamente procesos

hechos. históricos y se van nombrando diferentes acontecimientos. Se utilizan marcadores cualitativos y cuantitativos. Algunos utilizan línea de tiempo.

Fuente: Elaboración propia

Un 72\% (18) de estudiantes desarrolla un tiempo histórico lineal e incorpora en diferentes niveles la simultaneidad cronológica. Las categorías existentes son:

Tabla 9

Categorías de Simultaneidad

Vinculación $\quad$ La simultaneidad se expresa a través de un marcador cualitativo que generalizada y cualitativa permite vincular lo que ocurre en dos espacios diferentes, pero en un mismo tiempo. La vinculación es mencionada solo una vez en el relato y se desarrolla a través de una generalización. Los marcadores cualitativos que dan cuenta de esta categoría son: contexto internacional o extranjero, mundo.

Vinculación a La simultaneidad se expresa a través de la explicitación de hechos o través de procesos históricos que permiten vincular lo que ocurre en dos procesos o espacios diferentes, pero en un mismo tiempo. Sin datar hechos $\quad$ cuantitativamente el tiempo en que ocurren los procesos o hechos históricos, sin simultáneos.

datación.

Vinculación a La simultaneidad se expresa a través de la explicitación de hechos o través de procesos históricos que permiten vincular lo que ocurre en dos procesos o espacios diferentes, pero en un mismo tiempo datando hechos cuantitativamente al menos un proceso o hecho simultáneo.

históricos con

datación.

Fuente: Elaboración propia

La vinculación a través de procesos o hechos históricos, con datación, es la categoría menos presente con un 16\% (4). A15 explica el proceso de Guerra Fría y da cuenta de este tipo de simultaneidad de la siguiente forma:

El siglo XX en un principio se caracterizó por la existencia de conflictos y situaciones de crisis a nivel mundial. En este sentido Chile no quedo ajeno a estos procesos (...) en la primera década del siglo XX, Europa había conseguido cimentar su 
dominio político y económico a nivel mundial. Dentro de este dominio político se genera una pugna entre distintos países por el control de territorios, en este marco se originó la Primera Guerra Mundial, conflicto bélico internacional que se dio entre 1914 y 1918 (...) Esto es importante para la historia chilena, ya que esta situación de crisis planetaria lograría incrementar las utilidades de la exportación de Salitre (A15).

Un 28\% (7) de los estudiantes delimita temporalmente el inicio y el fin del período, proceso o acontecimiento que desarrollara en su clase. La delimitación se realiza de forma cuantitativa, cualitativa o mixta.

A13 relata el proceso de Liberalismo. Lo delimita de forma mixta:

(...) estructuración desde el ideario liberal y su difusión a principios del siglo XIX en Europa y América. En este sentido utilizaría y proyectaría un cuadro comparativo que permitiera evidenciar las principales diferencias entre la monarquía (régimen existente en un contexto anterior) y la república. (A13)

Un $84 \%$ (21) de la muestra utiliza marcadores cuantitativos y cualitativos en su relato, mientras que un 16\% (4) no incorpora ningún marcador cuantitativo. El 100\% de ellos si incorpora marcadores cualitativos que nos permiten situar el tiempo histórico del relato. Para Pagès (1989) es esencial establecer la duración de cada período o proceso, porque permite diferenciarlos del período o proceso anterior y posterior.

\section{Conclusiones}

Hemos abordado el presente artículo planteándonos varias interrogantes iniciales ¿qué habilidades de pensamiento histórico han desarrollado estudiantes de formación inicial?, ¿los estudiantes antes de cursar asignaturas de didáctica han desarrollado la mayoría de las habilidades del pensamiento histórico? y ¿están preparados los futuros docentes para desarrollar el pensamiento histórico en sus estudiantes? Preguntas que se entrelazan con el objetivo del artículo que consistió en evaluar las habilidades de pensamiento histórico y su enseñanza, que poseen los estudiantes de formación inicial al comenzar la asignatura de didáctica de la historia, particularmente la conciencia histórica temporal.

La conciencia histórica temporal permite situarnos en el tiempo presente, explicando la realidad actual a través del pasado y proyectándonos las múltiples opciones que tenemos para el futuro. En este contexto, la educación y sobre todo el aprendizaje de la historia nos ayudan a ser conscientes de esta articulación temporal. En este sentido cobra relevancia la presente investigación, puesto que nos permite extraer algunas conclusiones que pueden servir para mejorar, en el futuro, la enseñanza de la historia en los centros educativos y permitir que más jóvenes aprendan a pensar el tiempo.

Respecto a la pregunta ¿qué habilidades de pensamiento histórico han desarrollado estudiantes de formación inicial? A partir de la dimensión de conciencia histórica temporal, es posible concluir que:

- Los estudiantes incorporan las dimensiones temporales pasado y/o presente, sin caer en el presentismo.

- Incorporan de manera más recurrente el cambio en un 52\% que la continuidad en un $40 \%$.

- El tratamiento del tiempo histórico es lineal y se expresa de diversas formas. 
- La mayoría de ellos considera la simultaneidad cronológica.

- Utilizan marcadores temporales cuantitativos y cualitativos. Marcadores cuantitativos más frecuentes son siglos y años. Los cualitativos, el nombre de algún período histórico, sin embargo, no siempre va acompañado de la datación.

En relación con la segunda pregunta propuesta ¿los estudiantes de formación inicial antes de cursar asignaturas de didáctica han desarrollado la mayoría de las habilidades del pensamiento histórico? considerando que la muestra la constituyen estudiantes de quinto y séptimo semestre que están en la mitad de su carrera, es posible, responder que el pensamiento histórico se desarrolla a lo largo de la vida y que sus habilidades no se adquieren completamente en la universidad. Pero también hay que precisar que, al comenzar las asignaturas de didáctica de la historia, la herencia de pensamiento histórico, tiene algunas privaciones:

- El futuro es el aspecto menos desarrollado de la temporalidad. Si no incluimos el futuro ¿es posible que los estudiantes se sientan protagonistas de la historia? 0 ¿estamos promoviendo una educación efectiva para la ciudadanía y la democracia?

- Cuentan la historia desde el pasado, por lo que, falta establecer relaciones temporales, que permitan explicar el presente a través del pasado y proyectarnos al futuro.

- Un 16\% de los estudiantes no incorpora ningún marcador temporal cuantitativo e incorpora escasos marcadores cualitativos.

Considerando la última pregunta ¿están preparados los futuros docentes para desarrollar el pensamiento histórico en sus estudiantes? A la luz de la investigación, creemos que tienen habilidades que han desarrollado a lo largo de su vida académica, pero que estas habilidades se deben seguir fortaleciendo desde la formación inicial docente, generando puntos de encuentro y de diálogo entre las diferentes disciplinas que conforman los curriculum de los centros universitarios, fundamentalmente la didáctica y la historia, para el caso del pensamiento histórico.

\section{Referencias bibliográficas}

Braudel, F. (1950) Las responsabilidades de la historia. Lección inaugural, leída el viernes 01 de diciembre de 1950 en Collége de France, Cátedra de Historia de la Civilización moderna. En BRAUDEL, F (1974) La Historia y las Ciencias Sociales. Madrid: Editorial Alianza.

Braudel, F. (1959) L'Apport de l'Historie des Civilisations. Encyclopidie Francaise, Tomo XX, Le Monde en devenir, 20 págs. Capítulo V. En BRAUDEL, F (1974) La Historia y las Ciencias Sociales. Madrid: Editorial Alianza.

Burke, P. (1993) (ed.) Formas de hacer Historia. Madrid: Editorial Alianza.

Carr, E. (1978). ¿Qué es la historia? España: Editorial Seix Barral.

Chávez, C y Pagés, J. (2017). Didáctica de la historia y enseñanza del pensamiento histórico en formación inicial de docentes de secundaria en Chile. En Martínez, R. (coord.), García-Morís, R. (coord.) y García, C. (coord.), Investigación en didáctica de las ciencias sociales. Retos preguntas y líneas de investigación (pp. 73-82). España: Universidad de Córdoba.

De Certeau, M. (2006) La escritura de la historia. México: Universidad Iberoamericana. Instituto tecnológico de estudios superiores de occidente. 
Domínguez, J. (1989) El lugar de la Historia en el currículum 11-16. Un marco general de referencia. En Carretero M, Pozo J y Asensio M. La enseñanza de las ciencias sociales (pp. 3360). Madrid: Aprendizaje Visor.

Domínguez, J. (2015) Pensamiento histórico y evaluación de competencias. Barcelona: Editorial Graó.

Fontana, J. (2013) Historia: Análisis del pasado y proyecto social. España: Editorial Austral. Primera edición 1982.

Heller, A. (1982). Teoría de la Historia. Barcelona: Editorial Fontamara.

Hernández, R., Fernández, C. y Baptista, P. (2003). Metodología de la Investigación. México: Editorial Mc Graw Hill Educación.

MINEDUC. (2015) Bases curriculares de $7^{\circ}$ a $2^{\circ}$ medio. Chile. Recuperado en: https://media.mineduc.cl/wp-content/uploads/sites/28/2017/07/Bases-Curriculares-

7o-básico-a-2º-medio.pdf

Pagès, J. (1988). Situar-se en el temps, situar-se en la història. Guix, 124, 11-16

Pagès, J. (1989). Aproximación a un currículum sobre el tiempo histórico. En Rodríguez Frutos, J. (ed.). Enseñar historia. Nuevas propuestas (pp. 107-138). Barcelona: Laia/Cuadernos de Pedagogía.

Pagès, J. (2012a). Conciencia y Tiempo Histórico. Perspectiva Escolar. Monografías, 1, 35-40.

Pagès, J. (2012b) Enseñar la actualidad ¿Para qué? Perspectiva Escolar. Monografías, 1, 80-88.

Pagès, J. y Santisteban, A (2018) La enseñanza de la Historia. Historia y Memoria, 17, Juliodiciembre, 11-16.

Pagès, J. y Santisteban, A. (1999) La enseñanza del tiempo histórico: una propuesta para superar viejos problemas. En García, T (Coord) Curriculum de las ciencias sociales para el siglo XXI, que contenidos y para qué (pp. 187-207). España: Díada Editorial.

Pagès, J. y Santisteban, A. (2010) La enseñanza y el aprendizaje del tiempo histórico en la educación primeria. Cuadernos CEDES, 30, (82), 281-309.

Rüsen, J. (1992) El libro de texto ideal. Consideraciones acerca del medio de orientación en la enseñanza histórica. Ponencia presentada en la Segunda reunión argentino-alemana sobre libros de texto. Georg Eckert, Institut, Braunschweig mayo de 1992. Publicado en Finocchio, S. (1993). Enseñar Ciencias Sociales. Buenos Aires: Editorial Troquel.

Rüsen, J. (2007). Memory, history and the quest for the future, in Cajani, L. (Ed.). History Teaching, Identities and Citizenship. European Issues in Children's Identity and Citizenship, 7, CiCe. Stoke on Trent. Trentham Books.

Santisteban, A (2010). La formación de competencias de pensamiento histórico. Memoria Académica, 14, 34-56.

Santisteban, A. (2007) Una investigación sobre cómo se aprende a enseñar el tiempo histórico. Enseñanza de las Ciencias Sociales, 6, 19-29.

Santisteban, A. (2012). Tiempo al tiempo en la escuela: una mirada transversal. Perspectiva Escolar. Monografías, 1, 43-48.

Santisteban, A. y Angueras, C. (2014). Formación de la conciencia histórica y educación para el futuro. Clío \& Asociados. 18-19, 249-267. 
Santisteban, A., González, N. y Pagès, J. (2010). Una investigación sobre la formación del pensamiento histórico. En Ávila, R. Rivero, P y Domínguez, P. (coords.) Metodología de investigación en Didáctica de las Ciencias Sociales (pp. 115-128.) Zaragoza: Fernando el Católico -Diputación de Zaragoza/ AUPDCS.

Seixas, P. \& Ericikan, K. (Eds) (2015) New Directions in Assessing Historical Thinking. New York: Routledge

Seixas, P. \& Peck, C. (2004). Teaching historical thinking. In A. Sears \& I. Wright (Eds), Challengens and Prospects for Canadian Social Studies (pp. 109-117). Vancouver: Pacific Educational Press.

Seixas, P., Morton, T., Colyer, J., y Fornazzari, S. (2013). The big six: Historical thinking concepts. Toronto: Nelson Education

Simián de Molinas, S. (1970). El método retrospectivo en la enseñanza de la historia. Buenos Aires: Paidós.

Strauss, A y Corbin, J. (2002). Bases de la investigación cualitativa. Técnicas y procedimientos para desarrollar la teoría fundamentada. Medellín: Editorial Universidad de Antioquía.

Torres, P. (2001a). La enseñanza del tiempo histórico. Proyecto didáctico Quirón. Madrid: Ediciones de la Torre.

Torres, P. (2001b). Didáctica de la historia y educación de la temporalidad: Tiempo social y tiempo histórico. Madrid: Universidad Nacional de Educación a Distancia. 